\title{
Viral expression associated with gastrointestinal adenocarcinomas in TCGA high-throughput sequencing data
}

\author{
Daria Salyakina $^{1 *}$ and Nicholas F Tsinoremas ${ }^{1,2}$
}

\begin{abstract}
Background: Up to 20\% of cancers worldwide are thought to be associated with microbial pathogens, including bacteria and viruses. The widely used methods of viral infection detection are usually limited to a few a priori suspected viruses in one cancer type. To our knowledge, there have not been many broad screening approaches to address this problem more comprehensively.
\end{abstract}

Methods: In this study, we performed a comprehensive screening for viruses in nine common cancers using a multistep computational approach. Tumor transcriptome and genome sequencing data were available from The Cancer Genome Atlas (TCGA). Nine hundred fifty eight primary tumors in nine common cancers with poor prognosis were screened against a non-redundant database of virus sequences. DNA sequences from normal matched tissue specimens were used as controls to test whether each virus is associated with tumors.

Results: We identified human papilloma virus type 18 (HPV-18) and four human herpes viruses (HHV) types 4, 5, 6B, and 8, also known as EBV, CMV, roseola virus, and KSHV, in colon, rectal, and stomach adenocarcinomas. In total, 59\% of screened gastrointestinal adenocarcinomas (GIA) were positive for at least one virus: 26\% for EBV, 21\% for CMV, $7 \%$ for HHV-6B, and 20\% for HPV-18. Over 20\% of tumors were co-infected with multiple viruses. Two viruses (EBV and CMV) were statistically significantly associated with colorectal cancers when compared to the matched healthy tissues from the same individuals ( $p=0.02$ and 0.03 , respectively). HPV-18 was not detected in DNA, and thus, no association testing was possible. Nevertheless, HPV-18 expression patterns suggest viral integration in the host genome, consistent with the potentially oncogenic nature of HPV-18 in colorectal adenocarcinomas. The estimated counts of viral copies were below one per cell for all identified viruses and approached the detection limit.

Conclusions: Our comprehensive screening for viruses in multiple cancer types using next-generation sequencing data clearly demonstrates the presence of viral sequences in GIA. EBV, CMV, and HPV-18 are potentially causal for GIA, although their oncogenic role is yet to be established.

Keywords: Cancer, Papilloma virus, Herpes virus

\section{Background}

Viruses may be more commonly associated with malignant diseases than previously considered [1]. Reported associations do not always mean that a virus is a direct cause of the cancer; they can be the result of contamination, viral infection without causal involvement ('passenger'), and an indirect or direct causal relationship. Regardless of

\footnotetext{
* Correspondence: dsalyakina@med.miami.edu

${ }^{1}$ Center for Computational Science, University of Miami, 1120 NW 14 St, Miami, FL 33136, USA

Full list of author information is available at the end of the article
}

the causal relationship, viruses may have significant clinical implications in human cancers through contribution to dramatic changes in the microenvironment and immunosurveillance.

The main strategies to detect and type various viruses in cancers usually address individual protein biomarkers, serological tests, or DNA/RNA detection of one or a few viruses at a time. The major disadvantage of these strategies is failure to detect viruses not previously known to be associated with a particular cancer type. In this report, we introduce a new and substantially different

\section{Biomed Central}

(c) 2013 Salyakina and Tsinoremas; licensee BioMed Central Ltd. This is an open access article distributed under the terms of the Creative Commons Attribution License (http://creativecommons.org/licenses/by/2.0), which permits unrestricted use, distribution, and reproduction in any medium, provided the original work is properly cited. 
way of addressing this problem by utilizing next-generation sequencing (NGS) data to detect both human and nonhuman nucleic acids in tumor specimens. This approach does not require any prior knowledge of viruses involved and can identify all known viral genomes. NGS provides the opportunity to detect viral transcripts with high sensitivity in the host tissue at frequencies less than 1 RNA molecule in 1 million [2]. Whole genome or transcriptome tumor sequencing data provides a unique resource for the development of new and powerful methodologies to detect and characterize viruses in cancers.

'Computational subtraction' is the general concept for detecting infectious agents in the host NGS material [3]. During this procedure, human and artifact sequences are removed from the NGS data and the remaining sequences are aligned to bacterial or viral references from existing databases. A few groups have implemented computational subtraction procedures for this purpose $[4,5]$. To date, NGS data was successfully used for virus identification in human papilloma virus (HPV)-associated squamous cell carcinomas [6-9] and hepatitis B virus (HBV)-mediated hepatocellular carcinomas [5], while other cancer types and viruses largely remain out of the picture.

In this study, we present a comprehensive screening for viruses in NGS data of nine common cancers in 1,007 patients, using The Cancer Genome Atlas (TCGA) data. TCGA is a joint project of the National Cancer Institute (NCI) and the National Human Genome Research Institute (NHGRI). The goal of the TCGA project is to collect and systematically explore the entire spectrum of genomic changes involved in more than 20 types of human cancers. Comprehensive genomic characterization has been published for three out of nine cancers studied so far (lung squamous cell carcinoma, colon, and rectum adenocarcinoma) $[10,11]$. Colon and rectum adenocarcinoma were shown to belong to one cancer type based on their molecular profiles. In addition to the screening for viruses, we perform association analysis of identified viruses with tumor vs. paired non-malignant tissue from the same patients in order to determine whether the presence of a virus is significantly associated with tumors and not the normal cell types.

\section{Results and discussion}

\section{Screening for viruses}

Screening for viruses is an essential step in the continuum of research that is expected to lead to new treatment strategies in patients with virus-positive tumors. In this study, we performed the systematic screening for potentially oncogenic viruses in nine cancer types from TCGA (Table 1), most of which were not previously known to be associated with viruses or have controversial reports in this regard [12,13]. After subtraction of
Table 1 Sample size for available DNA and RNA sequencing data in primary tumors and paired control samples

\begin{tabular}{|c|c|c|c|c|}
\hline \multirow[t]{3}{*}{ Cohort } & \multirow{2}{*}{\multicolumn{2}{|c|}{$\begin{array}{l}\text { Primary } \\
\text { tumor }\end{array}$}} & \multicolumn{2}{|c|}{ Matched controls } \\
\hline & & & \multirow{2}{*}{$\begin{array}{l}\text { Blood } \\
\text { DNA }\end{array}$} & \multirow{2}{*}{$\begin{array}{c}\text { Solid tissue } \\
\text { DNA }\end{array}$} \\
\hline & RNA & DNA & & \\
\hline AML, acute myeloid leukemia & 123 & nt & nt & nt \\
\hline COAD, colon adenocarcinoma & 194 & 77 & 56 & 16 \\
\hline KIRK, kidney renal clear carcinoma & 132 & $\mathrm{nt}$ & $\mathrm{nt}$ & $\mathrm{nt}$ \\
\hline $\begin{array}{l}\text { KIRP, kidney renal papillary cell } \\
\text { carcinoma }\end{array}$ & 15 & $\mathrm{nt}$ & $\mathrm{nt}$ & $\mathrm{nt}$ \\
\hline LUAD, lung adenocarcinoma & 58 & $\mathrm{nt}$ & $\mathrm{nt}$ & $\mathrm{nt}$ \\
\hline LUSK, lung squamous cell carcinoma & 151 & nt & nt & nt \\
\hline READ, rectum adenocarcinoma & 71 & 40 & 35 & 4 \\
\hline STAD, stomach adenocarcinoma & 57 & 3 & 0 & 2 \\
\hline $\begin{array}{l}\text { UCEC, uterine corpus endometrioid } \\
\text { carcinoma }\end{array}$ & 157 & $\mathrm{nt}$ & $\mathrm{nt}$ & $\mathrm{nt}$ \\
\hline
\end{tabular}

Control samples were derived from either whole blood or adjacent normal tissue (not both) from the same patient. nt, not tested.

non-viral sequences, unaligned fragments were used in virus identification (Figure 1). Sequences of Epstein-Barr virus (EBV), cytomegalovirus (CMV), roseola virus (HHV-6B), Kaposi's sarcoma-associated human virus (KSHV), and human papilloma virus type 18 (HPV-18) were identified in transcriptomes of three gastrointestinal adenocarcinomas (GIA): stomach, rectum, and colon adenocarcinomas (STAD, READ, and COAD, respectively). In total, $83 \%$ of the viral reads mapped to the known coding regions. The remaining six cancer types (Table 1 ) were virus negative according to our computational pipeline. Table 2 represents a summary for the sequencing data in the GIA samples and matched controls.

\section{Viruses in gastrointestinal adenocarcinomas}

EBV, CMV, and HHV-6B were detected in all three GIA, while HPV-18 was detected in colorectal cancers only. In total, 189 (58.7\%) GIA samples were infected with at least one virus: 83 (25.8\%) with EBV, 67 (20.8\%) with CMV, 22 (6.8\%) with HHV-6B, and 64 (19.8\%) with HPV-18 (Figure 2). One STAD RNA sample was KSHV positive with 115 sequence reads mapping to 3,280 bases of the viral genome (Additional file 1: Table S1). During the review of this paper, another group published a very similar study including 3,775 tumors from TCGA [14]. However, in their computational pipeline, Khoury with colleagues did not detect any viral sequences in 138 COAD and 66 READ samples. In addition, they only reported four EBV-positive samples in the STAD. Khoury et al. used a different sequence aligner (MOSAIK) and more stringent cutoff for the viral gene expression detection. Although no explicit cutoff is stated in the paper 


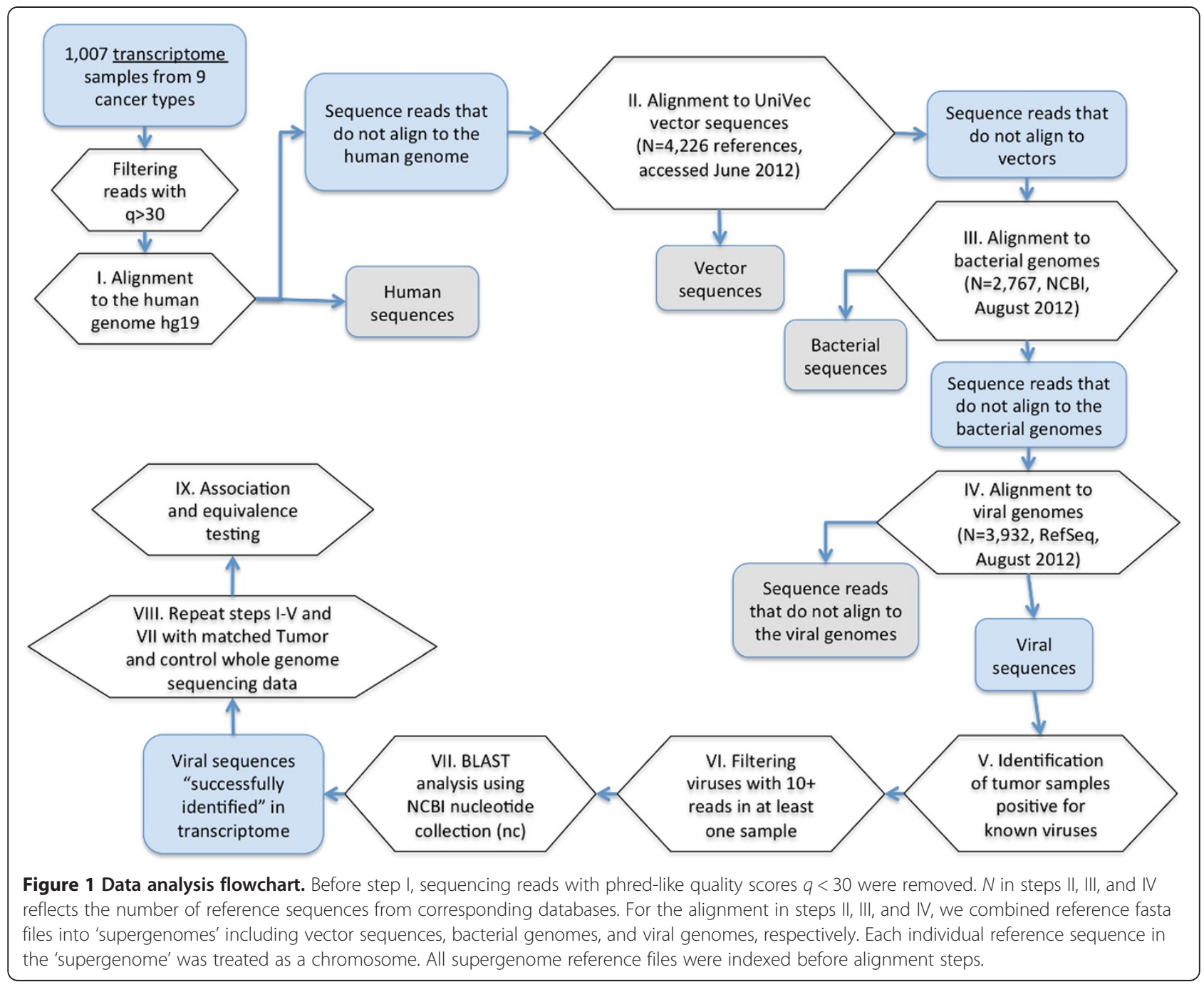

[14], the VirusSeq pipeline [5] implemented in their study uses 1,000 reads as a cutoff for virus detection. In our case, the number of identified reads was far below 1,000 for all transcriptomes, except two EBV-positive STAD tumors. The probable reason for using such a high cutoff in VirusSeq pipeline is the high number of false-positive results due to the homology of the contaminating vector and bacterial sequences. In our pipeline, adding steps II and III, in which these sequences are subtracted, alleviates this problem, reducing the number of unaligned RNA-seq reads by $19 \%$ on average before aligning to the virus reference. In our experience, these two steps are essential since they eliminate falsepositive findings and increase specificity of the method. We included here 57 STAD samples, not 71 as in [14]. To our knowledge, 14 additional patients reported in [14] have withdrawn their consent to participate in TCGA study (as of November 1, 2012).

All viruses identified in GIA are ubiquitous in human population. The first encounter with EBV, CMV, and
HHV-6B infection usually happens in early childhood and results in latent lifelong infection with a prevalence of over $90 \%$ in adults [15-21]. Similarly, KSHV infects $7.2 \%$ and $49 \%$ of the population depending on the geographical region [22-24]. At the same time, HPV is the most common sexually transmitted infection with a lifelong prevalence of $71 \%$, although unlike herpes virus infections, about $90 \%$ of HPV infection is cleared within 2 years without any consequences [25].

EBV, HPV-18, KSHV, and CMV have been linked to multiple cancers and potentially are oncogenic in GIA. EBV has been accepted as an infective agent of gastric and colorectal carcinomas. Nearly $10 \%$ of all gastric cancers [26-30] and up to $30 \%$ of colorectal carcinomas [31-35] have been found to be EBV-infected monoclonal epithelial cells. In TCGA, $45.6 \%$ of STAD, $22.6 \%$ of COAD, and $31 \%$ of READ samples were EBV positive [36,37] (Figure 2). Multiple studies have also shown significant association of high-risk HPV types with colorectal cancers with infection rate of up to $84 \%$ [38-41]. The 
Table 2 Total number of next-generation sequencing reads/fragments available for gastrointestinal cancers organized by cancer and tissue type

\begin{tabular}{|c|c|c|c|c|c|c|c|c|}
\hline \multirow[t]{2}{*}{ Cohort } & \multirow[t]{2}{*}{ Statistics } & \multirow{2}{*}{$\begin{array}{c}\text { Primary tumor } \\
\text { RNA }\end{array}$} & \multicolumn{2}{|c|}{ Primary tumor } & \multicolumn{2}{|c|}{ Blood } & \multicolumn{2}{|c|}{ Solid tissue } \\
\hline & & & DNA & $\begin{array}{c}\text { Human genome } \\
\text { coverage }^{a}\end{array}$ & DNA & $\begin{array}{c}\text { Human genome } \\
\text { coverage }^{\mathrm{a}}\end{array}$ & DNA & $\begin{array}{c}\text { Human genome } \\
\text { coverage }^{a}\end{array}$ \\
\hline \multirow[t]{4}{*}{ COAD } & Min & $5,244,743$ & $60,872,080$ & 0.47 & $64,637,478$ & 0.50 & $148,341,394$ & 1.18 \\
\hline & Mean & $26,127,635$ & $205,217,167$ & 1.63 & $182,959,915$ & 1.45 & $224,838,391$ & 1.77 \\
\hline & Median & $27,424,762$ & $196,408,988$ & 1.57 & $193,657,350$ & 1.49 & $196,072,876$ & 1.57 \\
\hline & Max & $64,021,517$ & $1,162,469,344$ & 9.43 & $354,790,740$ & 2.85 & $429,443,164$ & 3.36 \\
\hline \multirow[t]{4}{*}{ READ } & Min & $19,665,974$ & $89,943,982$ & 0.70 & $90,122,736$ & 0.70 & $95,061,666$ & 0.75 \\
\hline & Mean & $26,782,703$ & $225,818,442$ & 1.79 & $214,787,544$ & 1.71 & $150,549,985$ & 1.19 \\
\hline & Median & $27,121,702$ & $227,226,239$ & 1.81 & $224,045,358$ & 1.80 & $159,027,517$ & 1.26 \\
\hline & Max & $33,338,326$ & $461,153,444$ & 3.68 & $367,295,986$ & 2.93 & $189,083,240$ & 1.47 \\
\hline \multirow[t]{4}{*}{ STAD } & Min & $127,157,036$ & $259,701,502$ & 2.08 & NA & NA & $418,702,808$ & 3.34 \\
\hline & Mean & $155,138,810$ & $300,668,630$ & 2.40 & NA & NA & $448,318,606$ & 3.58 \\
\hline & Median & $155,490,548$ & $280,015,346$ & 2.23 & NA & NA & $448,318,606$ & 3.58 \\
\hline & Max & $193,445,130$ & $362,289,042$ & 2.88 & NA & NA & $477,934,404$ & 3.82 \\
\hline
\end{tabular}

Paired reads are counted as two single fragments. COAD, colon adenocarcinoma; READ, rectum adenocarcinoma; STAD, stomach adenocarcinoma; NA, no data available. ${ }^{a}$ Average coverage was calculated as the number of reads multiplied by the average read length ( $\left.51 \mathrm{nt}\right)$ and divided by the corresponding genome length, divided by two for diploid genome.

detected frequency of HPV-18 in TCGA was substantially lower: $31 \%$ in COAD and $0.4 \%$ in READ. Differences in frequencies between previously reported and our studies can result from multiple factors, such as varying sensitivity of detection methods used as well as population demographics. For instance, EBV positivity in STAD has been reported to be higher in males, young subjects, non-antral subsites, diffuse-type histology, and in studies from the Americas [27]. Given the sample size of the STAD cohort and the number of viruses, no epidemiological analyses were done. COAD and READ combined provided a sufficient sample size for association testing with clinical and demographic variables (Additional file 1: Tables S2-S12). No association with gender, age at initial diagnosis, histological type or ' $\mathrm{M}$ ' and ' $\mathrm{N}$ ' staging was found. Nominal $p$ values are shown in Table 3. After correction for multiple testing, the only association of HPV-18 infection with anatomic subdivision was statistically significant. HPV-18 was predominantly associated with tumors located in the cecum and ascending colon. No large epidemiological studies for $\mathrm{HPV}$-associated COAD are available thus far to compare these results to.

CMV is also capable of transforming mammalian cells through various pathways [42] and has been linked to colorectal cancer, although available evidence is scarce [43]. KSHV causes Kaposi's sarcoma [44] and, to our knowledge, has not yet been associated with gastric adenocarcinomas. This may be a special case of STAD that needs further investigation.

\section{Co-infection with multiple viruses}

A substantial proportion of GIA was co-infected with multiple viruses: 46 (23\%) COAD, 11 (19.3\%) STAD, and

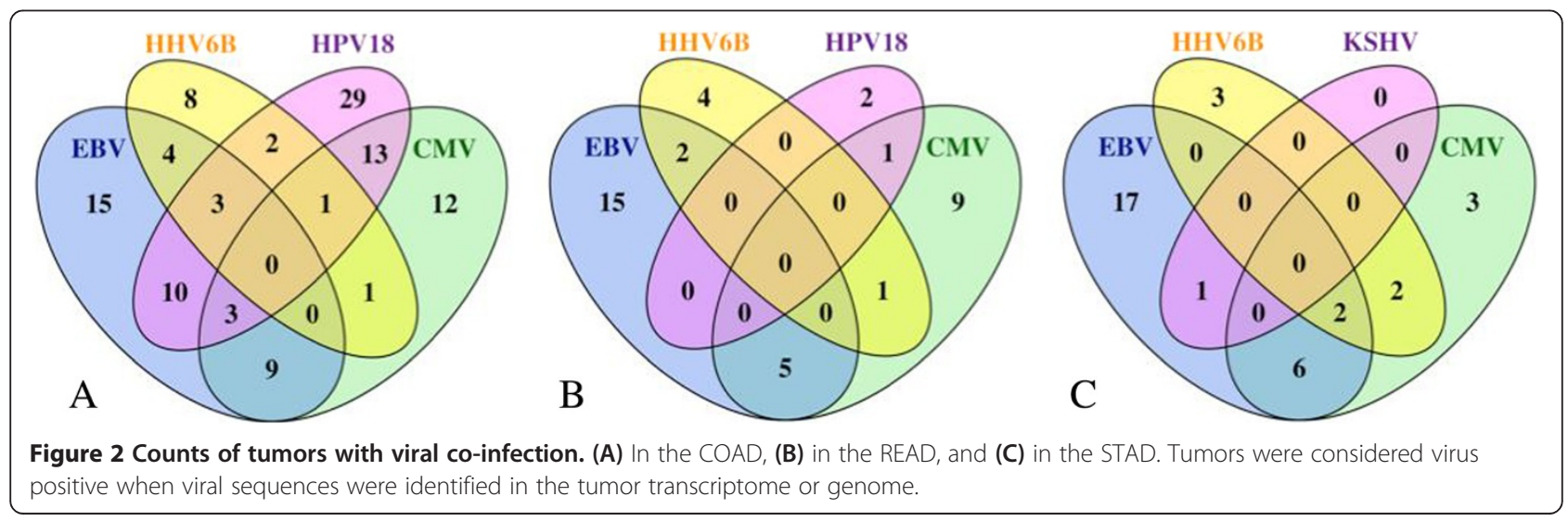




\begin{tabular}{|c|c|c|c|c|}
\hline Phenotype & HPV-18 & EBV & CMV & HHV-6B \\
\hline Gender & 1.00 & 1.00 & 1.00 & 0.54 \\
\hline Age at initial diagnosis & 0.22 & 0.51 & 0.07 & 0.26 \\
\hline Anatomic subdivision & $5.0 \mathrm{E}-05^{*}$ & 0.23 & 0.06 & 0.02 \\
\hline Histological type & 0.39 & 0.52 & 0.82 & 0.55 \\
\hline History of colon polyps & $3.0 \mathrm{E}-03$ & 0.77 & 0.22 & 0.83 \\
\hline Pathologic M & 0.84 & 0.32 & 0.21 & 1.00 \\
\hline Pathologic N & 0.59 & 0.11 & 0.58 & 0.13 \\
\hline Pathologic T & 0.33 & 0.03 & 0.09 & 0.48 \\
\hline Stage & 0.89 & 0.01 & 0.12 & 0.64 \\
\hline
\end{tabular}

${ }^{*} p$ value is significant after Bonferroni correction at $a=0.05$.

9 (12.7\%) READ. Figure 2 shows how many tumors were co-infected. These multiple viruses may either co-exist in the same cancer cells or populate different cell types that compose or infiltrate the tumor. Szostek et al. suggests that co-infection with $\mathrm{HHVs}$, especially CMV and EBV, may increase probability of the HPV-16 integration into the host genome during cervical cancer tumorigenesis [45]. Similar mechanisms may be involved with EBV, CMV, and HPV-18 in colorectal adenocarcinomas and need to be tested in future studies. Alternatively, some of the identified viruses can also preferentially infect cancer cells, taking advantage of the impaired immune environment of the tumors.

\section{Virus quantification}

The proportion of viral transcriptome reads relative to the total number of reads in the GIA ranged from $6 \times 10^{-9}$ (HHV-6B) to $2 \times 10^{-5}$ (EBV). The majority of tumors did not have a sufficient number of reads to cover the whole viral genome or to allow transcript quantification (see Additional file 1: Table S1 and Additional file 2). The number of viral sequence reads detected in tumor transcriptomes varied from a single fragment to tens of thousands of fragments per sample, with a strong skew towards low counts (Figure 3). Only five or less viral sequence fragments were detected in $49 \%$ of the transcriptomes and only one fragment in $21 \%$. However, our follow-up Basic Local Alignment Search Tool (BLAST) analysis, described in the 'Methods' section, demonstrated that even detection of a single short read may be sufficient to ensure unambiguous viral detection, confirming high specificity of sequencing alignment [8].

The estimated viral load for EBV, CMV, and HHV-6B in GIA was less than one viral copy per cell (vc/c) in all cases with a maximum of $0.72 \mathrm{vc} / \mathrm{c}(\mathrm{HHV}-6 \mathrm{~B})$ (Additional file 1: Table S1). The latter is equivalent to one viral genome per 1.39 human cells. This data supports the hypothesis that only a small proportion of tumor cells had a virus. The viral DNA abundance for EBV and CMV correlated with the proportion of total viral RNA reads (Additional file 3). Because no genomic data was available for KSHV-positive tumor and no HPV-18 was detectable in genomic DNA from tumors or normal cells, no viral load for these viruses could be calculated. The HPV-18 genome (7,857 nt for RefSeq ID: NC_001357) is 20 to 30 times smaller than the HHV (162,114-235,646 nt). The HPV-18 DNA quantity must have been below the detection limit at the available sequencing depth. The lowest detection threshold for NGS studies is limited to one sequence read aligning to the target genome. As a result, the probability for detecting a viral sequence in the host NGS data will be proportional to the target sequence length, the viral load, and the sequencing depth. Given the HPV-18 size and average genome sequencing depth of GIA, the hypothetical average detection limit would be above $0.0047 \mathrm{vc} / \mathrm{c}$ (standard deviation $(S D)=0.0023)$, equivalent to 1 virus in $261 \mathrm{hu}$ man cells $(\mathrm{SD}=133)$. This estimation does not take into account possible data loss due to disproportional filtering of highly polymorphic viral sequences through an unaccepted number of mismatches and homopolymeric and repetitive regions. Thus, the viral load is most likely underestimated here. Currently, there is no clear consensus on the minimum viral load indicative for the virus causality in neoplasm. On one hand, viral genome abundance and active expression of viral oncogenes are broadly believed to indicate much greater viral involvement in disease than the silent presence of viral genome. On the other hand, according to the 'hit-and-run' mechanism, transient acquisition of viral genome may be sufficient to induce malignant conversion [46]. In the hit-and-run scenario, viruses may get partially or completely lost after they cause permanent damage to the host cell and are no longer necessary for the maintenance of the malignant state. The most reliable estimates of viral load from the literature are related to HPV-associated tumors. The quantitative PCR experiments report several HPV-18 copies per cell $[8,47]$. However, Yoshida et al. suggested that very early HPV-18 DNA integration may result in lower copy numbers in cervical adenosquamous carcinoma (1.50$0.89 \mathrm{vc} / \mathrm{c}$ ), leading to a more aggressive transformation with greater chromosomal instabilities, higher growth rates, and rapid progression [47].

\section{Virus association with tumors}

Next, we tested if identified viruses were mostly found in tumors compared to the non-malignant matched tissue from the same individuals. One of the limitations of this study is the absence of transcriptome data for matched non-malignant tissues. Before association analysis, we explored if transcriptome and genome sequencing data can be pooled for tumors and compared to genomic data from non-malignant tissues. According to 

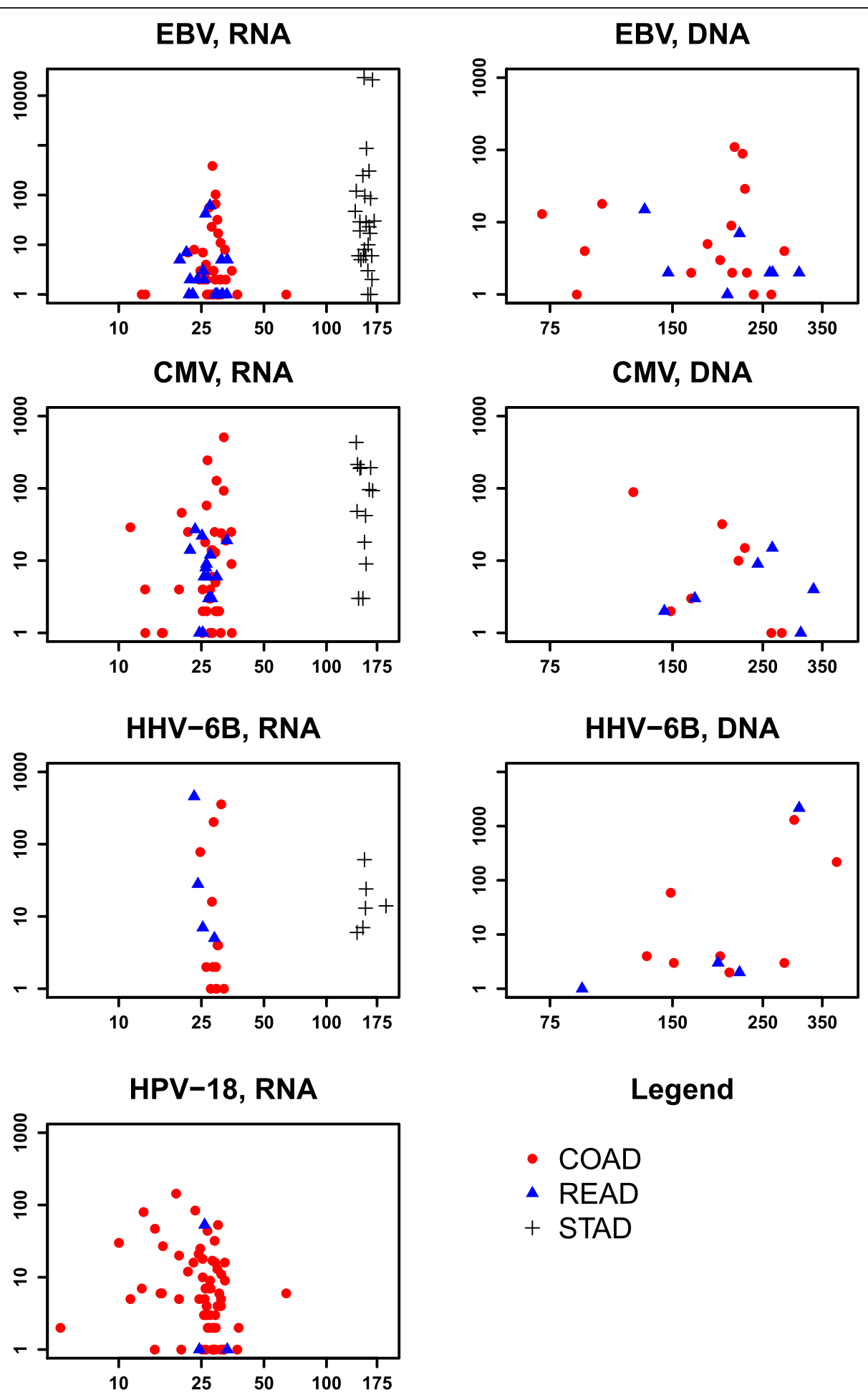

- COAD
- READ
+ STAD

Figure 3 Number of sequencing reads mapped to virus reference versus the total number of reads. The $X$-axis shows total RNA or DNA fragments (short sequencing reads) in millions, and the $Y$-axis shows the number of fragments mapped to the particular virus, as stated in the plot subtitles. Each data point represents one tumor. HPV-18 DNA was not detectable in the whole genome sequencing data. COAD are depicted as read circles, READ as blue triangles, and STAD as black plus signs. 
our results, tumor DNA and RNA did not show statistically equivalent rates of virus positivity (Table 4). For this reason, only DNA data from both tumors and nonmalignant matched controls were utilized for association analysis. A sufficient number of whole genome sequencing data was available for three viruses: EBV, CMV, and HHV-6B in COAD and READ only. Since COAD and READ are genetically identical [11], we combined the two into colorectal adenocarcinomas (CRAD) to achieve a higher sample size for association analysis. Our results indicate that EBV and CMV were significantly associated with CRAD vs. matched non-malignant specimens with $p=0.0022 \quad\left(p_{\text {corrected }}=0.02\right)$ and $p=0.0034 \quad\left(p_{\text {corrected }}\right.$ $=0.03$ ), respectively (Table 5 ). Since testing was done in three tissue types: solid tumor, solid non-malignant adjacent tissue, and whole blood, these associations may reflect tissue-specific infection. Thus, we investigated if viral rates differ between tissue types in controls. All controls positive for EBV and CMV were blood samples, and none of the tested solid tissue controls had these two viruses (Table 5). In contrary, HHV-6B was identified predominantly $(80 \%)$ in solid non-malignant tissue and not blood, although association was not significant after correction for multiple testing $\left(p_{\text {corrected }}=0.07\right)$. These findings may reflect the fact that EBV and CMV are associated with tumors, while $\mathrm{HHV}-6 \mathrm{~B}$ is associated with the colorectal tissue. EBV, CMV, and HPV-18 are capable to infect different types of skin cells, endothelium, or mucus membranes and are plausible infections in the histological context of GIA. While the oncogenic nature of CMV is still debatable [42], EBV and HPV-18 are potentially causal in GIA because both viruses encode oncoproteins, which are able to transform human cells $[30,39,48]$. HHV-6B is known to propagate preferentially in T lymphocytes or glial cells [49] and does not have any known oncogenes. The presence of HHV-6B in the colon and rectum may possibly originate from tissue infiltration by infected lymphocytes, although this hypothesis needs to be verified. One of the limitations of our study is the lack of availability of corresponding samples for further validation.

\section{Virus integration into the human genome}

Since no viral DNA for HPV-18 was identifiable, detection of integration sites of HPV-18 into the human genome was not possible. Nevertheless, the cumulative expression pattern of HPV-18 in CRAD supports evidence for genomic integration. The HPV episome usually becomes integrated into the host cell DNA during oncogenesis by opening the ring molecule and disrupting the $E 2$ gene, which normally suppresses oncogenes $E 6$ and E7. As a result, part of E2 and $L 2$ and whole $E 4$ and $E 5$ genes become deleted. Expression of E6 and E7 downregulates p53 and pRb and promotes malignancy
Table 4 Counts of colorectal samples, positive (+) or negative (-) for identified viruses, within tumor's DNA/RNA pairs

\begin{tabular}{cccccc}
\hline Sample type & Virus name & $\begin{array}{c}\text { Tumor DNA } \\
(\boldsymbol{N}=\mathbf{1 1 7})\end{array}$ & $\begin{array}{c}\text { Cl for two one-sided } \\
\text { hypotheses }(\mathrm{CL}=\mathbf{0 . 9 7 5})\end{array}$ \\
\hline Tumor RNA & EBV & + & - & $0 ; 3.09$ & $0.40 ;$ infinity \\
& + & 15 & 9 & & \\
- & 10 & 83 & & \\
CMV & + & - & $0 ; 0.51^{*}$ & 0.01 ; infinity \\
+ & 13 & 13 & & \\
& - & 1 & 90 & & \\
HHV-6B & + & - & $0 ; 18.84$ & 0.85 ; infinity \\
+ & 2 & 3 & & \\
& - & 10 & 102 & & \\
HPV-18 & + & - & $0 ; 0.14^{*}$ & $0 ;$ infinity \\
+ & 0 & 28 & & \\
- & 0 & 89 & & \\
\hline
\end{tabular}

$N$, number of matched pairs. *proportions are not equivalent at $a=0.05$ after Bonferroni correction.

[50]. While three early viral genes, E1, E6, and E7, showed high 'cumulative expression levels' in CRAD, partial expression of E2 and whole L2, E4 and E5 was not detected (Figure 4). Lack of expression in presumably deleted regions suggests the potentially oncogenic

Table 5 Counts of colorectal samples, positive $(+)$ or negative (-) for identified viruses, in matched tumor/normal specimen pairs

\begin{tabular}{|c|c|c|c|c|c|c|}
\hline \multirow[t]{2}{*}{$\begin{array}{l}\text { Sample } \\
\text { type }\end{array}$} & \multirow[t]{2}{*}{$\begin{array}{l}\text { Virus } \\
\text { name }\end{array}$} & \multirow{2}{*}{\multicolumn{2}{|c|}{$\begin{array}{c}\text { Matched } \\
\text { control DNA } \\
N=111 \\
2 \text { (blood:solid) }\end{array}$}} & \multicolumn{3}{|c|}{$\begin{array}{c}\text { Adjusted } p \text { values for } \\
\text { association }\end{array}$} \\
\hline & & & & $\begin{array}{l}\text { Tumors } \\
\text { vs. all }\end{array}$ & $\begin{array}{c}\text { Tumors } \\
\text { vs. }\end{array}$ & $\begin{array}{l}\text { Tumors } \\
\text { vs. solid }\end{array}$ \\
\hline \multirow{12}{*}{$\begin{array}{l}\text { Tumor } \\
\text { DNA }\end{array}$} & EBV & + & - & 0.02 & 0.06 & 1 \\
\hline & + & $3(3: 0)$ & $21(19: 2)$ & & & \\
\hline & - & $5(5: 0)$ & $82(64: 18)$ & & & \\
\hline & CMV & + & - & 0.03 & 0.06 & 1 \\
\hline & + & 0 & $12(11: 1)$ & & & \\
\hline & - & $1(1: 0)$ & $98(79: 19)$ & & & \\
\hline & $H H V-6 B$ & + & - & 1 & 0.98 & 0.07 \\
\hline & + & $4(1: 3)$ & $8(8: 0)$ & & & \\
\hline & - & $10(2: 8)$ & 89 (80:9) & & & \\
\hline & HPV-18 & + & - & - & - & - \\
\hline & + & 0 & 0 & & & \\
\hline & - & 0 & 0 & & & \\
\hline
\end{tabular}

$N$, number of matched pairs. blood: solid, counts for the non-malignant samples by tissue type: whole blood and solid adjacent tissue, respectively. 


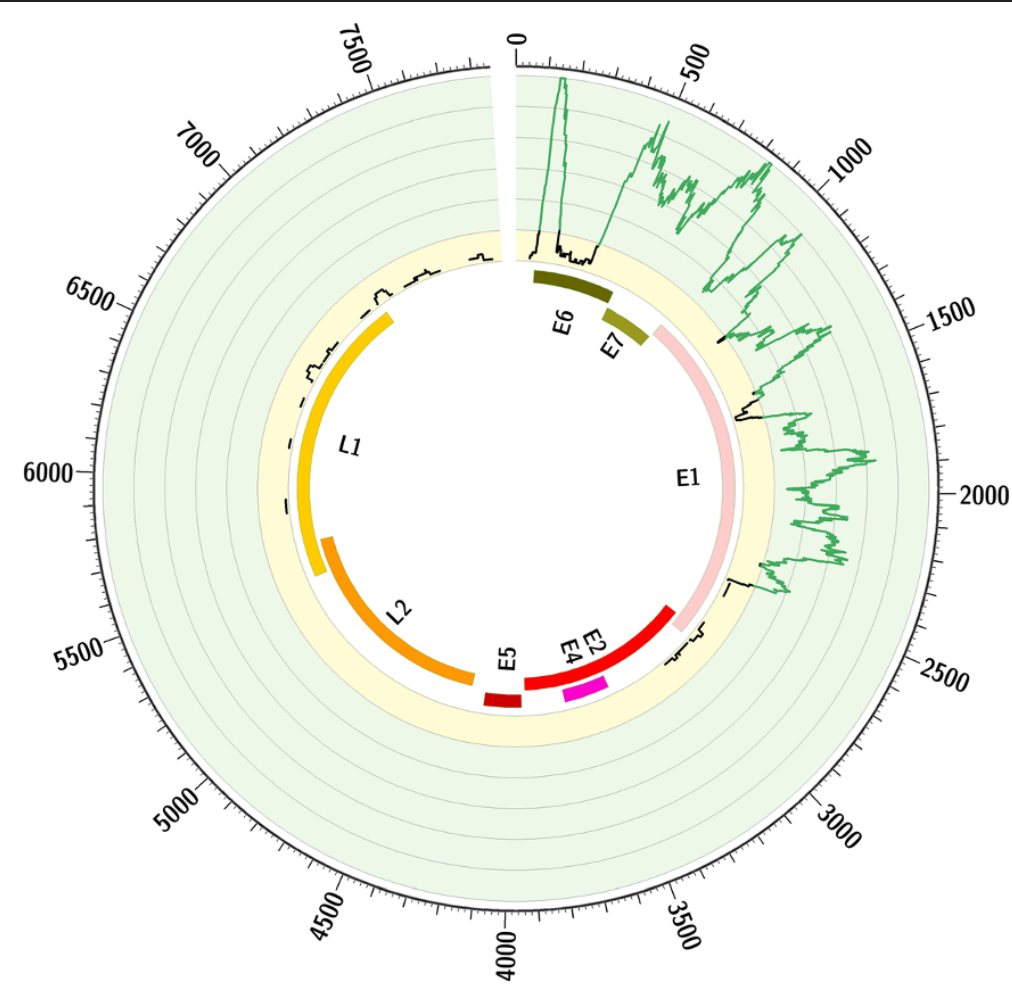

Figure 4 Circos plot for HPV-18 RNA-seq. From outside to the inside of the circle: (1) HPV-18 genomic positions; (2) number of sequencing reads mapped to the viral genome in 64 colon and rectum adenocarcinomas combined. Range from 1 to 10 shown on light yellow background as a black line, and from 11 to 61 on light green background as a green line; (3) viral genes mapped to genomic positions. While expression of genes $E 6, E 7$, and $E 1$ is obvious, genes $L 2, E 5, E 4$, and part of $E 2$ were not detected. This pattern is expected when HPV-18 integrates in the host genome and part of the viral genome becomes deleted.

nature of HPV-18. Despite collected evidence, the temporal relationship between infection and tumorigenesis cannot be disseminated from this data. To conclusively prove a causal role of viruses in cancer, a complete chain of evidence from epidemiology, histopathology, and molecular biology is required.

For the remaining viruses detected in available genomic data, the number of identified reads was not sufficient for integration site detection (see Figure 3 and Additional file 2). Neither viral nor human genomes were covered without substantial gaps. As shown in Table 2, the median coverage of the human genome in colorectal samples was below $2 x$, and a great majority of the viruses with available whole genome seq data had a small fraction of the genomes covered.

\section{Conclusions}

Our results clearly demonstrate the presence of viral sequences in GIA. EBV and CMV were statistically significantly associated with CRAD. In addition, the expression pattern of HPV-18 was consistent with genomic integration typical during oncogenesis [50]. This supports the hypothesis that EBV, CMV, and HPV-18 are potentially oncogenic in GIA, although we realize that further studies are needed before a conclusion can be made about the pathophysiological role of the identified viruses in GIA. No viruses were identified in the remaining six cancer types.

Our results demonstrate the feasibility of NGS for the identification of viruses at very low levels in the human tissue. Unlike PCR-based approach, NGS data offer a unique opportunity to capture any viral nucleic acids present in the sample above the detection limit. Identification of viral infection is a first step in determining the role of viruses in cancer. Availability of comprehensive viral databases makes it possible to scan for a large number of candidates without the need for de novo assembly with the restriction that novel viruses will not be detected.

Finally, in this study, we established an empirical detection limit in our computational pipeline. This information can be used to calculate the required sequencing depth, as well as the amount of material needed by the given size of viral genome, and the expected viral load in similar studies. 


\section{Methods}

\section{Subjects}

Whole transcriptome sequencing data for nine cancer types, comprising 1,007 patients, were obtained through TCGA (accessed on October 17, 2011). Table 1 summarizes specimen counts and abbreviations for the nine included cancer types. Additional transcriptome and whole genome sequencing data for three GIA were downloaded on November 1, 2012. Sequencing has been done using Illumina (Solexa, GAII, Illumina, Inc., San Diego, CA, USA) or SOLiD ${ }^{\mathrm{mm}}$ technology (Life Technologies, Carlsbad, CA, USA). A detailed description of the TCGA data can be found on the following TCGA websites: http:// cancergenome.nih.gov/, https://tcga-data.nci.nih.gov/tcga/, as well as in two recently published studies on genomic characterization of three out of nine cancer types discussed here $[10,11]$. Patient enrollment and utilization of data were conducted in accordance with TCGA human subjects protection and data access policies (http://cancergenome.nih.gov/PublishedContent/Files/pdfs/ 6.3.1_TCGA_Human_Subjects_and_Data_Access_policies_ FINAL_011211.pdf).

Cancers selected for the TCGA study were chosen based on specific criteria that included (1) poor prognosis and high public health impact, and (2) availability of human tumor and matched normal tissue that meet TCGA standards for patient consent, quality, and quantity. The proportion of $60 \%$ tumor nuclei in the specimens was found to be sufficient by TCGA project organizers to generate high-quality data, in which the tumor's signal can be distinguished from other cells' signals when using NGS. Only primary, untreated tumors were collected. Samples were frozen quickly after surgery in order to prevent degradation of the RNA and DNA.

Whole genome sequencing data was available for $37.5 \%$ of the GIA (Table 1). Blood or germ line specimens derived from the same individual as the tumor specimens were used in the TCGA study to serve as paired normal controls when available (Table 1). DNA sequencing data from the blood or adjacent healthy tissue was available for $35 \%$ of the GIA specimens.

\section{Bioinformatics analysis}

Transcriptome data (BAM files) generated by TCGA for a total of 1,007 cancer specimens were analyzed in an automated fashion on a computational cluster hosted by the High-Performance Computing core at the Center for Computational Science, University of Miami (http://ccs. miami.edu/). An IBM BladeCenter cluster was available for compute-intensive data analysis. The cluster, named Pegasus running under Linux operation system, was used consisting of 280 computing nodes each with 8 Xeon $2.6 \mathrm{GHz}$ cores and $16 \mathrm{~GB}$ of memory, and 700 computing nodes each with 4 Opteron $2.2 \mathrm{GHz}$ core and $4 \mathrm{~GB}$ of memory. These nodes are interconnected by Gigabit Ethernet and feature a 21 TB NFS file system providing an aggregate of 5,040 cores and 7.3 TB of memory. All computational tasks were submitted in parallel to the LSF job scheduler and resource management system. The computational pipeline is outlined in Figure 1. In total, 1,156 jobs were submitted to the cluster for steps I-V for DNA-seq and RNA-seq data; 25,245 CPU hours were used for data analysis. Bamtools-1.0.2 [51] and samtools-0.1.18 [52] software were employed for converting data format. Sequencing reads with phred-like quality scores $q>30$ were utilized. TopHat (v.2.0.0) [53] was consistently used for all transcriptome mapping steps. Multiple threads were used during alignment with option $-\mathrm{p} 8$. When subtracting bacterial and viral sequences, we allowed TopHat to tolerate up to four mismatches per read, instead of the default of two in the alignment step, to allow for potentially higher mismatch rates due to mutations [54] or imperfect match to the reference sequence. In addition, TopHat was instructed to use a gtf file and not to look for novel transcript junctions by utilizing the '-nonovel-junc' flag. We combined reference fasta files into 'supergenomes' for vector sequences, bacterial genomes, and viral genomes for steps II, III, and IV, respectively (Figure 1). Each individual reference sequence in the 'supergenome' was treated as a chromosome. Reference files were indexed before alignment steps. The bacterial reference file had to be split into two parts to reduce the memory use needed for indexing and mapping steps. Even the single short read aligning to the viral reference was considered as successful detection, if following BLAST analysis [55] versus the NCBI nucleotide (nt) collection confirmed sequence similarity with the target over $98 \%$ and at least one transcriptome out of a cohort had more than 10 reads mapped to a viral genome reference. Sequences aligning to multiple organisms, known artificial (vector) sequences, or low-complexity sequences were considered false positive and removed.

Only three gastrointestinal cancer types, stomach (STAD), rectum (READ), and colon adenocarcinomas (COAD), which tested virus positive on the transcriptome level, proceeded to the whole genome analysis step (Table 1). Burrows-Wheeler Aligner (BWA, v.0.5.9) [56] with default options was consistently used for genomic data alignment. Multiple threads were used when running BWA with option -t 4. Genomic read subtraction was performed in exactly the same fashion as described above for transcriptomes (Figure 1). In order to determine computational pipeline sensitivity to single nucleotide mismatches, sequence reads on EBV and HPV-18 with different mutation/mismatch rates and lengths were simulated and run through the computational subtraction and alignment steps (see Additional files 1 and 4). 


\section{Consensus sequence for EBV}

EBV reference genomes include two very similar strains HHV-4.1 (NC_007605) and HHV-4.2 (NC_009334). In order to capture as many reads as possible, we created a consensus sequence using both NC_007605 and NC_009334 genome references. We replaced the two original references for two strains with the consensus reference for computational pipeline.

\section{Statistical analysis}

Since available data comprised cases-only DNA/RNA and malignant/normal tissue pairs, a simple McNemar test implemented in R library 'exact2x2' (v1.1-1.0) was used for both equivalence and association testing [57]. Equivalence testing for virus identification in DNA and RNA was performed using two one-sided exact $\mathrm{McNe}$ mar tests with a confidence level of 0.975 at $\alpha=0.05$ (Bonferroni-corrected for four viruses). The null hypothesis of equivalence was rejected, when at least in one of the one-sided tests, the confidence interval (CI) did not include ' 1 '. Association of virus with tumor vs. normal tissue was done with two-sided exact McNemar test. Since COAD and READ are genetically identical [11], we combined two cohorts for association analysis to achieve a higher sample size. Association testing of virus presence in tumor/normal tissue was done using blood and solid tissue controls in separate tests, as well as blood and solid combined. Bonferroni correction was done for nine tests (three types of control groups multiplied by three viruses identified in the whole genome data). Fisher exact test was used for association testing with clinicopathological and demographics variables. Age at initial diagnosis in virus-positive and virusnegative groups was compared using ANOVA. Bonferroni correction was applied.

\section{Estimation of viral load}

We calculated $V$, the number of viral copies per cell (vc/c), i.e., viral load, for each DNA sample as

$$
V=C_{\mathrm{V}} / C_{\mathrm{H}}
$$

where $C_{\mathrm{V}}$ is the average sequencing coverage for the virus, and $C_{\mathrm{H}}$ is the average human genome coverage. Average coverage $C$ was calculated as

$$
C_{i}=R_{i} \times L / G_{i},
$$

where $i$ is the species, $R$ is the number of reads, $L=51$ and is the average read length in nucleotides, and $G$ is the corresponding genome length in nucleotides. For the diploid human genome, $C=C_{\mathrm{H}} / 2$.

\section{Additional files}

Additional file 1: Simulations and viral rate estimations.

A document containing simulation methods and results, and supplementary Tables 1-12, showing summary on viral reads detected, as well as clinical and demographic data in gastrointestinal adenocarcinomas.

Additional file 2: Dot matrix. This dot matrix view shows regions of similarity based upon the BLASTN 2.2.27+ results. The viral genome positions are on the $X$-axis. The lines represent mapped reads. The $Y$-axis shows cumulative bases of the aligned reads over all GIA sorted by the percentage of the genome covered. Higher coverage yields longer lines on the plot. Viral RNA transcriptome reads, when mapped to their reference genome sequences, showed uneven distribution clustering most likely corresponding to actively transcribed genes. Genomic reads, as expected, mapped along the viral reference genome randomly and more uniformly than transcriptomic reads.

Additional file 3: Correlation of EBV and CMV load in tumor's transcriptomes and genomes. Each data point represents one tumor. The $X$-axis shows log10-transformed percentage of viral reads in tumor's transcriptome; the $Y$-axis shows log10-transformed viral load ( $\mathrm{nc} / \mathrm{c}$, see methods) in tumor's whole genome. COAD are depicted as read circles, READ as blue triangles. STAD is not shown because there was not a sufficient number of tumor genomes sequenced.

Additional file 4: Simulation results. At the low mutation rate up to $2 \%$, derived reads were not lost to any significant extent, and our pipeline still captured over $94 \%$ simulated reads by BWA (whole genome pipeline) and over $80 \%$ by Tophat v.2.0.0 (transcriptome pipeline). Our approach had highest sensitivity with the shortest reads (50 nt), being at least $80 \%$ for BWA at a mismatch rate of 0.04 , and 0.05 for Tophat. Higher mutation rates greatly impacted sensitivity, especially for the longer sequence reads, consistent with the BWA and TopHat algorithms. TopHat used the bowtie2 aligner, which seems to be affected to a greater extent by the length of the reads, probably due to using a fixed number of mismatches $(N=4)$, while BWA allows a floating error rate $k$ depending on the read length. Simulated errors were randomly distributed. The longer the read, the more likely was the inclusion of mismatches. Subtraction of non-viral reads did not affect HPV-18 alignment, and less than $1 \%$ of CMV reads were lost through this process at zero mutation rate. As expected, computational subtraction eliminates individual viral sequences to various extents, depending on the degree of homology with non-viral reference sequences included in the filters.

\section{Abbreviations}

AML: Acute myeloid leukemia; Cl: Confidence interval; CMV: Cytomegalovirus; COAD: Colon adenocarcinoma; CRAD: Colorectal adenocarcinomas;

EBV: Epstein-Barr virus; GIA: Gastrointestinal adenocarcinomas; HHV: Human herpes virus; HHV-6B: Roseola virus; HPV-18: Human papilloma virus type 18; KIRK: Kidney renal clear carcinoma; KIRP: Kidney renal papillary cell carcinoma; KSHV: Kaposi's sarcoma-associated human virus; LUAD: Lung adenocarcinoma; LUSK: Lung squamous cell carcinoma; NGS: Nextgeneration sequencing; nt: Nucleotide; READ: Rectum adenocarcinoma; SD: Standard deviation; STAD: Stomach adenocarcinoma; TCGA: The Cancer Genome Atlas; UCEC: Uterine corpus endometrioid carcinoma; vc/c: Viral copies per cell.

\section{Competing interests}

The authors declare that they have no competing interests.

\section{Authors' contributions}

DS has designed and tested the analytical pipeline, downloaded and analyzed the data, and performed results interpretation and manuscript writing. NT has been involved in drafting and revising the manuscript and has contributed to the analytical pipeline design. Both authors have given final approval of the version to be published.

\section{Acknowledgements}

We would like to thank TCGA project organizers as well as all study participants. This work was supported by the Center for Computational 
Science (CCS), University of Miami and partially by a grant (1R03CA17105201A1) from National Cancer Institute ( $\mathrm{NCl}$ ). We also thank the HighPerformance Computing team at the Center for Computational Science, University of Miami, especially Joel P. Zysman, John Baringer, Pedro Davila, and Zongjun Hu for technical support, as well as Dr. Enrique A. Mesri (Microbiology and Immunology Department) and Dr. Jennifer Clarke (Epidemiology Department) for their intellectual contribution to the manuscript. Finally, we would also like to thank Camilo Valdes (CCS) for assistance with the simulations.

\section{Author details}

${ }^{1}$ Center for Computational Science, University of Miami, 1120 NW 14 St, Miami, FL 33136, USA. ${ }^{2}$ Department of Medicine, Miller School of Medicine, University of Miami, 1120 NW 14 St, Miami, FL 33136, USA.

Received: 21 August 2013 Accepted: 7 November 2013

Published: 27 November 2013

\section{References}

1. Zur Hausen $\mathrm{H}$ : The search for infectious causes of human cancers: where and why. Virology 2009, 392(1):1-10.

2. Moore RA, Warren RL, Freeman JD, Gustavsen JA, Chenard C, Friedman JM, Suttle CA, Zhao YJ, Holt RA: The sensitivity of massively parallel sequencing for detecting candidate infectious agents associated with human tissue. PLoS One 2011, 6(5):e19838.

3. Weber G, Shendure J, Tanenbaum DM, Church GM, Meyerson M: Identification of foreign gene sequences by transcript filtering against the human genome. Nature genetics 2002, 30(2):141-142.

4. Kostic AD, Ojesina Al, Pedamallu CS, Jung J, Verhaak RG, Getz G, Meyerson M: PathSeq: software to identify or discover microbes by deep sequencing of human tissue. Nature biotechnology 2011, 29(5):393-396.

5. Chen Y, Yao H, Thompson EJ, Tannir NM, Weinstein JN, Su X: VirusSeq: software to identify viruses and their integration sites using nextgeneration sequencing of human cancer tissue. Bioinformatics 2013, 29(2):266-267.

6. Agrawal N, Frederick MJ, Pickering CR, Bettegowda C, Chang K, Li RJ, Fakhry C, Xie TX, Zhang J, Wang J, et al: Exome sequencing of head and neck squamous cell carcinoma reveals inactivating mutations in NOTCH1. Science 2011, 333(6046):1154-1157.

7. Stransky N, Egloff AM, Tward AD, Kostic AD, Cibulskis K, Sivachenko A, Kryukov GV, Lawrence MS, Sougnez C, McKenna A, et al: The mutational landscape of head and neck squamous cell carcinoma. Science 2011, 333(6046)::1157-1160.

8. Arron ST, Ruby JG, Dybbro E, Ganem D, DeRisi JL: Transcriptome sequencing demonstrates that human papillomavirus is not active in cutaneous squamous cell carcinoma. J Invest Dermatol 2011 , 131(8):1745-1753.

9. Barzon L, Militello V, Lavezzo E, Franchin E, Peta E, Squarzon L, Trevisan M, Pagni S, Dal Bello F, Toppo S, Palù G: Human papillomavirus genotyping by 454 next generation sequencing technology. J Clin Virol 2011, 52(2):93-97.

10. TCGARN: Comprehensive genomic characterization of squamous cell lung cancers. Nature 2012, 489(7417):519-525.

11. TCGARN: Comprehensive molecular characterization of human colon and rectal cancer. Nature 2012, 487(7407):330-337.

12. De Paoli P, Carbone A: Carcinogenic viruses and solid cancers without sufficient evidence of causal association. International Journal of Cancer Journal International du Cancer 2013, 133(7):1517-1529.

13. Grulich AE, van Leeuwen MT, Falster MO, Vajdic CM: Incidence of cancers in people with HIV/AIDS compared with immunosuppressed transplant recipients: a meta-analysis. Lancet 2007, 370(9581):59-67.

14. Khoury JD, Tannir NM, Williams MD, Chen Y, Yao H, Zhang J, Thompson EJ, Meric-Bernstam F, Medeiros $L J$, Weinstein JN, Su X: Landscape of DNA virus associations across human malignant cancers: analysis of 3,775 cases using RNA-Seq. Journal of Virology 2013, 87(16):8916-8926.

15. Takeuchi K, Tanaka-Taya K, Kazuyama Y, Ito YM, Hashimoto S, Fukayama M, Mori S: Prevalence of Epstein-Barr virus in Japan: trends and future prediction. Pathol Int 2006, 56(3):112-116.

16. Linton MS, Kroeker K, Fedorak D, Dieleman L, Fedorak RN: Prevalence of Epstein-Barr Virus in a population of patients with inflammatory bowel disease: a prospective cohort study. Aliment Pharmacol Ther 2013, 38:1248-1254

17. Luzuriaga K, Sullivan JL: Infectious mononucleosis. The New England Journal of Medicine 2010, 362(21):1993-2000.

18. Lubeck PR, Doerr HW, Rabenau HF: Epidemiology of human cytomegalovirus (HCMV) in an urban region of Germany: what has changed? Med Microbiol Immunol 2010, 199(1):53-60.

19. Lopo S, Vinagre E, Palminha P, Paixao MT, Nogueira P, Freitas MG Seroprevalence to cytomegalovirus in the Portuguese population, 2002-2003. Euro Surveill 2011, 16(25):5.

20. Staras SA, Dollard SC, Radford KW, Flanders WD, Pass RF, Cannon MJ Seroprevalence of cytomegalovirus infection in the United States, 1988-1994. Clin Infect Dis 2006, 43(9):1143-1151.

21. Oren I, Sobel JD: Human herpesvirus type 6: review. Clin Infect Dis 1992, 14(3):741-746.

22. Butler LM, Were WA, Balinandi S, Downing R, Dollard S, Neilands TB, Gupta S, Rutherford GW, Mermin J: Human herpesvirus 8 infection in children and adults in a population-based study in rural Uganda. The Journal of Infectious Diseases 2011, 203(5):625-634.

23. Qu L, Jenkins F, Triulzi DJ: Human herpesvirus 8 genomes and seroprevalence in United States blood donors. Transfusion 2010, 50 (5):1050-1056

24. Baillargeon J, Leach CT, Deng JH, Gao SJ, Jenson HB: High prevalence of human herpesvirus 8 (HHV-8) infection in south Texas children. Journal of Medical Virology 2002, 67(4):542-548.

25. CDC: Incidence, Prevalence, and Cost of Sexually Transmitted Infections in the United States. Atlanta: Centers for Disease Control and Prevention; 2013.

26. Uozaki H, Fukayama M: Epstein-Barr virus and gastric carcinoma-viral carcinogenesis through epigenetic mechanisms. Int I Clin Exp Pathol 2008, 1(3):198-216.

27. Camargo MC, Murphy G, Koriyama C, Pfeiffer RM, Kim WH, Herrera-Goepfert R, Corvalan AH, Carrascal E, Abdirad A, Anwar M, Hao Z, Kattoor J, Yoshiwara-Wakabayashi E, Eizuru Y, Rabkin CS, Akiba S: Determinants of Epstein-Barr virus-positive gastric cancer: an international pooled analysis. British Journal of Cancer 2011, 105(1):38-43.

28. Fukayama M, Ushiku T: Epstein-Barr virus-associated gastric carcinoma. Pathol Res Pract 2011, 207(9):529-537.

29. Marquitz AR, Mathur A, Shair KH, Raab-Traub N: Infection of Epstein-Barr virus in a gastric carcinoma cell line induces anchorage independence and global changes in gene expression. Proc Natl Acad Sci U S A 2012, 109(24):9593-9598.

30. Ryan JL, Jones RJ, Kenney SC, Rivenbark AG, Tang W, Knight ER, Coleman WB, Gulley ML: Epstein-Barr virus-specific methylation of human genes in gastric cancer cells. Infect Agent Cancer 2010, 5:27.

31. Yuen ST, Chung LP, Leung SY, Luk IS, Chan SY, Ho J: In situ detection of Epstein-Barr virus in gastric and colorectal adenocarcinomas. The American Journal of Surgical Pathology 1994, 18(11):1158-1163.

32. Grinstein S, Preciado MV, Gattuso P, Chabay PA, Warren WH, De Matteo E, Gould VE: Demonstration of Epstein-Barr virus in carcinomas of various sites. Cancer Research 2002, 62(17):4876-4878.

33. Karpinski P, Myszka A, Ramsey D, Kielan W, Sasiadek MM: Detection of vira DNA sequences in sporadic colorectal cancers in relation to $\mathrm{CpG}$ island methylation and methylator phenotype. Tumour Biol 2011, 32(4):653-659.

34. Park JM, Choi MG, Kim SW, Chung IS, Yang CW, Kim YS, Jung CK, Lee KY, Kang $\mathrm{JH}$ : Increased incidence of colorectal malignancies in renal transplant recipients: a case control study. Am J Transplant 2010, 10 (9):2043-2050.

35. Song LB, Zhang X, Zhang CQ, Zhang Y, Pan ZZ, Liao WT, Li MZ, Zeng MS: Infection of Epstein-Barr virus in colorectal cancer in Chinese. Ai Zheng 2006, 25(11):1356-1360.

36. Giuliani L, Ronci C, Bonifacio D, Di Bonito L, Favalli C, Perno CF, Syrjanen K, Ciotti M: Detection of oncogenic DNA viruses in colorectal cancer. Anticancer Res 2008, 28(2B):1405-1410

37. Chen TH, Huang CC, Yeh KT, Chang SH, Chang SW, Sung WW, Cheng YW, Lee H: Human papilloma virus 16 E6 oncoprotein associated with p53 inactivation in colorectal cancer. World J Gastroenterol 2012, 18(30):4051-4058.

38. Salepci T, Yazici H, Dane F, Topuz E, Dalay N, Onat H, Aykan F, Seker M, Aydiner A: Detection of human papillomavirus DNA by polymerase chain reaction and southern blot hybridization in colorectal cancer patients. J Buon 2009, 14(3):495-499. 
39. Lee YM, Leu SY, Chiang H, Fung CP, Liu WT: Human papillomavirus type 18 in colorectal cancer. Journal of Microbiology, Immunology, and Infection = Wei mian yu gan ran za zhi 2001, 34(2):87-91.

40. Bodaghi S, Yamanegi K, Xiao SY, Da Costa M, Palefsky JM, Zheng ZM: Colorectal papillomavirus infection in patients with colorectal cancer. Clinical Cancer Research 2005, 11(8):2862-2867.

41. Damin DC, Caetano MB, Rosito MA, Schwartsmann G, Damin AS, Frazzon $A P$, Ruppenthal RD, Alexandre CO: Evidence for an association of human papillomavirus infection and colorectal cancer. Eur J Surg Oncol 2007, 33(5):569-574.

42. Collins D, Hogan AM, Winter DC: Microbial and viral pathogens in colorectal cancer. The Lancet Oncology 2011, 12(5):504-512.

43. Chen HP, Jiang JK, Chen CY, Chou TY, Chen YC, Chang YT, Lin SF, Chan CH, Yang CY, Lin CH, Lin JK, Cho WL, Chan YJ: Human cytomegalovirus preferentially infects the neoplastic epithelium of colorectal cancer: a quantitative and histological analysis. J Clin Virol 2012, 54(3):240-244.

44. Zur Hausen H: Oncogenic DNA viruses. Oncogene 2001, 20(54):7820-7823.

45. Szostek S, Zawilinska B, Kopec J, Kosz-Vnenchak M: Herpesviruses as possible cofactors in HPV-16-related oncogenesis. Acta Biochim Pol 2009, 56 (2):337-342.

46. Niller $\mathrm{HH}$, Wolf $\mathrm{H}$, Minarovits J: Viral hit and run-oncogenesis: genetic and epigenetic scenarios. Cancer Letters 2011, 305(2):200-217.

47. Yoshida T, Sano T, Oyama T, Kanuma T, Fukuda T: Prevalence, viral load, and physical status of HPV 16 and 18 in cervical adenosquamous carcinoma. Virchows Arch 2009, 455(3):253-259.

48. Lee JH, Kim SH, Han SH, An JS, Lee ES, Kim YS: Clinicopathological and molecular characteristics of Epstein-Barr virus-associated gastric carcinoma: a meta-analysis. J Gastroenterol Hepatol 2009, 24(3):354-365.

49. Mori Y: Recent topics related to human herpesvirus 6 cell tropism. Cell Microbiol 2009, 11(7):1001-1006.

50. Zur Hausen $\mathrm{H}$ : Papillomaviruses and cancer: from basic studies to clinical application. Nature reviews Cancer 2002, 2(5):342-350.

51. Barnett DW, Garrison EK, Quinlan AR, Stromberg MP, Marth GT: BamTools: a $\mathrm{C}++\mathrm{API}$ and toolkit for analyzing and managing BAM files. Bioinformatics 2011, 27(12):1691-1692.

52. Li H, Handsaker B, Wysoker A, Fennell T, Ruan J, Homer N, Marth G, Abecasis G, Durbin R: The Sequence Alignment/Map format and SAMtools. Bioinformatics 2009, 25(16):2078-2079.

53. Trapnell C, Roberts A, Goff L, Pertea G, Kim D, Kelley DR, Pimentel H, Salzberg SL, Rinn JL, Pachter L: Differential gene and transcript expression analysis of RNA-seq experiments with TopHat and Cufflinks. Nat Protoc 2012, 7(3):562-578

54. Sanjuan R, Nebot MR, Chirico N, Mansky LM, Belshaw R: Viral mutation rates. Journal of virology 2010, 84(19):9733-9748.

55. Altschul SF, Madden TL, Schaffer AA, Zhang J, Zhang Z, Miller W, Lipman DJ: Gapped BLAST and PSI-BLAST: a new generation of protein database search programs. Nucleic Acids Res 1997, 25(17):3389-3402.

56. Li H, Durbin R: Fast and accurate short read alignment with BurrowsWheeler transform. Bioinformatics 2009, 25(14):1754-1760.

57. Fay MP: Confidence intervals that match Fisher's exact or Blaker's exact tests. Biostatistics 2010, 11(2):373-374.

doi:10.1186/1479-7364-7-23

Cite this article as: Salyakina and Tsinoremas: Viral expression associated with gastrointestinal adenocarcinomas in TCGA high-throughput sequencing data. Human Genomics 2013 7:23.

\section{Submit your next manuscript to BioMed Central and take full advantage of:}

- Convenient online submission

- Thorough peer review

- No space constraints or color figure charges

- Immediate publication on acceptance

- Inclusion in PubMed, CAS, Scopus and Google Scholar

- Research which is freely available for redistribution

Submit your manuscript at www.biomedcentral.com/submit
C Biomed Central 